\title{
Effects of convection on the removal of the multiplicity of stable states in one-dimensional vertical models
}

\author{
By TONI PUJOL* \\ Department of Atmospheric Sciences, Texas Agricultural and Mechanical University, USA
}

(Received 4 July 2002; revised 22 September 2003)

\section{SUMMARY}

Here I develop a model of a radiative-convective atmosphere with both radiative and convective schemes highly simplified. The atmospheric absorption of radiation at selective wavelengths makes use of constant mass absorption coefficients in finite width spectral bands. The convective regime is introduced by using a prescribed lapse rate in the troposphere. The main novelty of the radiative-convective model developed here is that it is solved without using any angular approximation for the radiation field. The solution obtained in the purely radiation mode (i.e. with convection ignored) leads to multiple equilibria of stable states, being very similar to some results recently found in simple models of planetary atmospheres. However, the introduction of convective processes removes the multiple equilibria of stable states. This shows the importance of taking convective processes into account even for qualitative analyses of planetary atmospheres.

KeYwords: Climatic states Multiple equilibria Radiative-convective models Radiative transfer

\section{INTRODUCTION}

Recently, a variety of simple one-dimensional (1D) purely radiative equilibrium models have been used to study the global sensitivity of planetary atmospheres, finding a multiplicity of stable states (i.e. distinct vertical temperature profiles that emit the same outgoing long-wave radiation (OLR)) induced by different mechanisms. For example, Lorenz et al. (1999) and Pujol and Fort (2002) obtain multiple equilibria of stable states in a grey atmosphere (i.e. an atmosphere with absorption of long-wave radiation independent of wavelength), with atmospheric absorption of short-wave radiation dependent on the long-wave optical thickness. A similar result is found by Pujol and North (2002, 2003) in a semi-grey atmosphere (i.e. an atmosphere with constant mass absorption coefficients in finite width infrared bands) transparent to sunlight. Li et al. (1997) and Ide et al. (2001), among others, have also found a multiplicity of stable states in simple pure radiative 1D vertical models. Note that such a multiple solution implies a hysteresis cycle on the mean planetary temperature as a function of the incoming short-wave radiation that may have important implications in the long-term evolution of several planetary atmospheres (see e.g. Lorenz et al. 1999).

The qualitative solution obtained from pure radiative equilibrium atmospheres (as in the studies cited above) was not expected to differ excessively from the actual radiative-convective atmosphere (e.g. in the number of stable states, in the qualitative response to changes in both external and internal parameters, etc.). The purpose of the present paper is to show the importance of taking non-radiative processes into account in the analysis of the global mean climate, and to investigate how the convective processes may vary the results obtained from pure radiative equilibrium atmospheres. Note that my aim is not to analyse the differences between pure radiative and radiativeconvective solutions found for the earth's present atmospheric conditions (see e.g. the classical and excellent discussions in Manabe and Möller (1961); Manabe and Strickler (1964) and Manabe and Wetherald (1967)). Rather than being interested in the solution for one particular scenario of one particular atmosphere, my aim is to examine more

* Corresponding address: Departament de Física, Escola Politècnica Superior, Universitat de Girona, Campus Montilivi, 17071 Girona, Catalonia, Spain. e-mail: taller@transpujol.com

(C) Royal Meteorological Society, 2004. 
generally the differences in the behaviour of radiative and radiative-convective planetary atmospheres, even though the cases analysed may not be of interest for the earth's current conditions.

The rationale of the paper is as follows. First, I show that a purely radiative equilibrium model that simulates the mean standard atmosphere leads to multiple equilibria of stable steady states. Then, I show how this multiplicity of stable states disappears when convective processes are included in the model atmosphere. From the above, and in order to reveal the actual nature of the climate system, I suggest a reanalysis of those studies that lead to multiple stable steady states in pure radiative equilibrium atmospheres by taking non-radiative processes into account.

The model atmosphere used here is described in detail in section 2; it improves the Radiative-Convective Model (RCM) employed in Pujol and North (2002) by ignoring any angular approximation for the radiation field. The novelty of such a treatment of the radiation field in comparison with a variety of previous simple 1D models (as in e.g. Nakajima et al. 1992; Pujol and North 2002, 2003) consists in an application of the energy balance condition panchromatically (as it should be) and not monochromatically. However, this improvement of the physical description of the processes removes the possibility of deducing analytical or semi-analytical solutions. In essence, the model atmosphere is of intermediate complexity for the radiative processes, being of very low complexity for the convective ones.

The model atmosphere developed in section 2 is evaluated in section 3 for the earth's current conditions in order to tune its free parameters and to check its global sensitivity. Note that this tuning process (unavoidable in simple climate models, see North et al. (1981)) gains in robustness if I use a climate state where I have reasonable knowledge of the sensitivity to changes in both external and internal parameters. This does not conflict with my main purpose stated above, since few changes in the model parameters lead to an entirely different atmosphere than that currently observed for the earth. Of course, section 3 would have been made redundant by using one of the many simple vertical climate models that may be found in the literature.

The results for different scenarios obtained in a pure radiative equilibrium version of the model atmosphere are shown in section 4. Here, multiple equilibria of stable steady states are found, being very similar in nature to those obtained by Pujol and Fort (2002) and Pujol and North $(2002 ; 2003)$. Section 4 also shows that such a multiplicity disappears when convection is included in the model atmosphere. As far as I know, this is the first time that the removal of stable states (i.e. of an entire branch of stable states) due to convection is shown.

Finally, in section 5 I emphasize the importance of including convective processes when analysing the mean standard atmosphere.

\section{ONE-DIMENSIONAL RADIATIVE-CONVECTIVE MODEL}

\section{(a) Radiative processes}

For simplicity in the 1D RCM I assume a plane parallel stratified atmosphere and neglect scattering processes. Then, the variation of the specific intensity of long-wave radiation $I_{\nu}$ (flux of energy in a given direction per second per unit frequency) that travels through an atmospheric slab of thickness $\mathrm{d} p, p$ being the atmospheric pressure, follows the radiative transfer equation:

$$
\mu \frac{\mathrm{d} I_{v}}{\mathrm{~d} p}=\frac{k_{v i} q_{i}}{g}\left(I_{\nu}-B_{v}\right),
$$


where $\mu$ is the cosine of the zenith angle, $k_{v i}$ is the mass absorption coefficient of the $i$ th absorbing gas at frequency $v, q_{i}$ is the specific mass of the $i$ th absorbing gas (mass of the $i$ th absorbing gas per unit mass of air), $g$ is the acceleration due to gravity, and $B_{v}$ is Planck's function. Equation (1) implicitly assumes local thermodynamic equilibrium conditions (LTE), from which the source function is equal to Planck's function. The hydrostatic equation has also been applied to (1) to use pressure as the vertical coordinate. The term $k_{v i} q_{i}$ in (1) represents a sum over the $i$ gases, allowing the existence of overlapping absorption bands.

Following Goody and Yung (1989), the heat gained by matter from long-wave radiation in unit frequency $h_{v}\left(\mathrm{~W} \mathrm{~m}^{-3}\right.$ per unit frequency) reads:

$$
h_{v}=4 \pi k_{v i} q_{i} \rho\left(\bar{I}_{v}-B_{v}\right)
$$

where $\rho$ is the air density, and $\bar{I}_{v}$ is the specific intensity of $I_{v}$ averaged over all directions.

$$
\bar{I}_{v}(p)=\frac{1}{2} \int_{0}^{1}\left\{I_{v}(p, \mu)+I_{v}(p,-\mu)\right\} \mathrm{d} \mu,
$$

where $I_{\nu}(p, \mu)$ and $I_{\nu}(p,-\mu)$ refer to intensities of upward (towards the top of atmosphere (TOA)) $(1 \geqslant \mu>0)$ and downward travelling beams $(1 \geqslant-\mu>0)$ at level $p$, respectively.

For infrared radiation, the boundary conditions are: zero downward radiation at TOA (i.e. $\left.I_{\nu}(0,-\mu)=0\right)$, and upward radiation equal to that of a black body at the surface (i.e. $I_{v}\left(p_{\mathrm{s}}, \mu\right)=B_{v}\left(T_{\mathrm{S}}\right)$, where $p_{\mathrm{s}}$ and $T_{\mathrm{S}}$ are the surface pressure and temperature, respectively). With these boundary conditions, the solution of (1) is:

$$
\begin{aligned}
I_{\nu}(p, \mu)= & B_{\nu}\left(T_{\mathrm{s}}\right) \exp \left(-\frac{1}{g \mu} \int_{p}^{p_{\mathrm{s}}} k_{\nu i} q_{i} \mathrm{~d} p\right) \\
& +\frac{1}{g \mu} \int_{p}^{p_{\mathrm{s}}} k_{\nu i} q_{i} B_{\nu} \exp \left(-\frac{1}{g \mu} \int_{p}^{p^{\prime}} k_{v i} q_{i} \mathrm{~d} p^{\prime \prime}\right) \mathrm{d} p^{\prime}, \quad(1 \geqslant \mu>0), \\
I_{\nu}(p,-\mu)= & \frac{1}{g \mu} \int_{0}^{p} k_{\nu i} q_{i} B_{\nu} \exp \left(-\frac{1}{g \mu} \int_{p^{\prime}}^{p} k_{\nu i} q_{i} \mathrm{~d} p^{\prime \prime}\right) \mathrm{d} p^{\prime}, \quad(1 \geqslant-\mu>0) .
\end{aligned}
$$

Water vapour and carbon dioxide are the only infrared-absorbing gases taken into account, whereas I assume that ozone absorbs short-wave radiation only (similar conditions to those used by Lindzen et al. 1982). In general the mass absorption coefficients of these gases are functions of temperature and pressure (see e.g. Vardavas and Carver 1984; Houghton 1986). Here the mass absorption coefficients, $k_{v i}$, are constant in finite width spectral bands (although the absorption within the atmospheric window by water dimers will be taken to be proportional to the water vapour partial pressure), which is quite realistic (see Kasting et al. 1984).

For simplicity, I assume a single infrared absorption band for carbon dioxide (ranging from 13 to $17.6 \mu \mathrm{m}$, Lindzen et al. (1982)), and a region (from 8 to $12 \mu \mathrm{m}$ ) of low absorption due to water vapour (atmospheric window). Then, I divide the infrared spectrum into five bands as shown in Fig. 1: bands 1, 3 and 5 use the mass absorption coefficient $k_{\mathrm{wv}}$ of water vapour outside the atmospheric window; band 2 uses the mass absorption coefficient $k_{\mathrm{wvw}}$ of water vapour inside the atmospheric window; and finally band 4 uses the mass absorption coefficients both of the water vapour outside the 


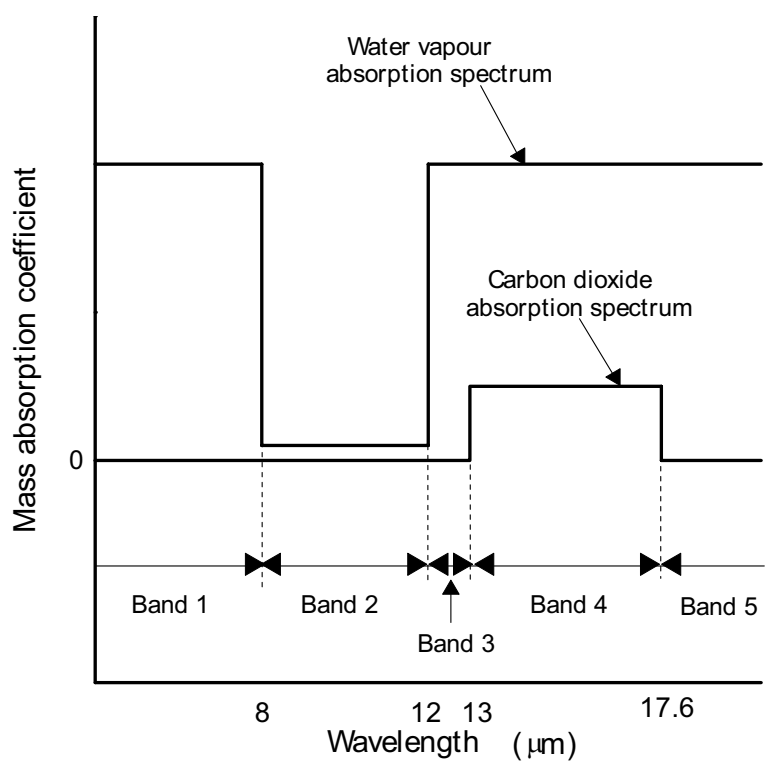

Figure 1. The semi-grey model uses five infrared bands with different but constant mass absorption coefficients. The limits of the carbon dioxide band follow from Lindzen et al. (1982). In some cases, we assume an absorption with the atmospheric window ( 8 to $12 \mu \mathrm{m}$ ) as a function of the partial pressure of water vapour. Grey atmospheres in the infrared spectrum use mass absorption coefficients independent of wavelength.

atmospheric window $k_{\mathrm{wv}}$ and of the carbon dioxide $k_{\mathrm{cd}}$. The free parameters $k_{\mathrm{wv}}, k_{\mathrm{wvw}}$ and $k_{\mathrm{cd}}$ will be tuned to produce the earth's current values of atmospheric opacity, and its expected climatic sensitivity to doubling the amount of carbon dioxide without any feedback (see section 3).

The specific mass of water vapour (specific humidity) $q_{\mathrm{wv}}$ varies with pressure and temperature and will be a function of the convective regime. Since carbon dioxide is a well-mixed gas, I assume a constant value of its specific mass $q_{\text {cd }}$, of $503 \times 10^{-6}$ (from the US Standard Atmosphere (1976); see Jursa (1985)), which is equivalent to 330 ppmv.

From Fig. 1, (2) can be integrated over each of the $m$ th (five) infrared spectral bands, giving the contribution of the $m$ th infrared band to the heat gained by matter from radiation $\left(\mathrm{W} \mathrm{m}^{-3}\right)$ :

$$
h_{m}=4 \pi k_{m i} q_{i} \rho\left(\bar{I}_{m}-\beta_{m} B\right),
$$

where

$$
\beta_{m}=\frac{1}{B} \int_{v_{m 1}}^{v_{m} \mathrm{u}} B_{v} \mathrm{~d} \nu .
$$

In (6), $B$ is the integral of Planck's function over all frequencies $\left(\propto T^{4}\right)$, and $v_{m 1}$ and $v_{m u}$ represent the lower and the upper frequency limits of the $m$ th band, respectively. $\beta_{m}$ will be referred to as the effective width of the $m$ th infrared band (note that $\sum_{m=1}^{5} \beta_{m}=1$ ) and, in general, $\beta_{m}$ is a function of temperature (see appendix). Equation (6) was first introduced by Weaver and Ramanathan (1995).

In (5) $\bar{I}_{m}$ is obtained from the integration of (3) over the frequency interval of the $m$ th infrared band, taking into account (4a) and (4b) and also that $k_{1 i} q_{i}=k_{3 i} q_{i}=$ 
$k_{5 i} q_{i} \equiv k_{\mathrm{wv}} q_{\mathrm{wv}}, k_{2 i} q_{i} \equiv k_{\mathrm{wvw}} q_{\mathrm{wv}}$ and $k_{4 i} q_{i} \equiv k_{\mathrm{wv}} q_{\mathrm{wv}}+k_{\mathrm{cd}} q_{\mathrm{cd}}$. The total heat per unit volume gained by matter due to infrared radiation is $h_{\mathrm{LW}}=\sum_{m=1}^{5} h_{m}$.

For simplicity, I use a simple analytical expression for the contribution of the shortwave absorption by ozone to the heating rate, $h_{\mathrm{SW}}$. Following Goody and Yung (1989):

$$
h_{\mathrm{SW}}(p)=S_{\mathrm{oz}}(0) k_{\mathrm{oz}} q_{\mathrm{oz}} \rho \exp \left(-\frac{1}{g \xi_{\mathrm{s}}} \int_{0}^{p} k_{\mathrm{oz}} q_{\mathrm{oz}} \mathrm{d} p^{\prime}\right),
$$

where $k_{\mathrm{oz}}$ is the ozone mass absorption coefficient (constant), $q_{\mathrm{oz}}$ is its specific mass, $\xi_{\mathrm{s}}$ is the absolute value of the cosine of the zenith angle of the solar beam $(=1 / 4)$, and $S_{\mathrm{oz}}(0)$ is the solar irradiance at TOA integrated over the ozone band. The dependence of $q_{\mathrm{oz}}$ on pressure is taken as:

$$
q_{\mathrm{oz}}(p)=q_{\mathrm{oz} m x} \exp \left\{-(\ln (p)-\ln (5))^{2} / 2 \sigma_{\mathrm{oz}}^{2}\right\},
$$

where $p$ is pressure (in $\mathrm{hPa}$ ) and $q_{\mathrm{ozm} x}=1.3 \times 10^{-5}$. Equation (8) with $\sigma_{\mathrm{oz}}=\sqrt{ } 2$ reasonably reproduces the vertical distribution of ozone given by the US Standard Atmosphere (1976) for atmospheric pressures higher than $0.1 \mathrm{hPa}$ (the region where the LTE equilibrium holds).

The vertical temperature profile in the stratosphere is obtained by assuming radiative equilibrium conditions (see appendix):

$$
h_{\mathrm{LW}}(p)+h_{\mathrm{SW}}(p)=0 .
$$

Note that the model developed here refers to clear-sky conditions. Further studies may include a simple cloud model with fractional cloud cover, cloud optical depth, cloud thickness and cloud-top height as the main variables describing cloud effects (see Vardavas and Carver 1984, 1985).

\section{(b) Convective adjustment}

The temperature profile in the troposphere (the region with convective processes, and with the earth's surface and the stratosphere as boundaries below and above it, respectively) is prescribed. Thus, I enforce the vertical temperature profile in the troposphere to follow a given lapse rate $\mathrm{d} T / \mathrm{d} z$ where $T$ is the air temperature and $z$ the altitude. For comparison, the implications of using three different lapse rates in the model atmosphere are analysed. The simplest adjustment of convective processes consists of using a constant lapse rate $\Gamma_{\mathrm{e}}$, equal to that for the mean standard atmosphere $\left(\mathrm{d} T / \mathrm{d} z=-\Gamma_{\mathrm{e}}\right.$, with $\Gamma_{\mathrm{e}}$ the environmental lapse rate equal to $\left.6.5 \mathrm{~K} \mathrm{~km}^{-1}\right)$. Since I use pressure as the vertical coordinate for carrying out the calculations, the tropospheric vertical temperature gradient in terms of pressure, when assuming constant $\Gamma_{\mathrm{e}}$, reads:

$$
\frac{\mathrm{d} T}{\mathrm{~d} p}=\frac{\Gamma_{\mathrm{e}}}{\rho g},
$$

where use has been made of the hydrostatic equation $(\mathrm{d} p=-\rho g \mathrm{~d} z)$.

I also analyse the results when assuming a moist adiabatic lapse rate for a pseudoadiabatic process. In this case, the tropospheric vertical temperature gradient in terms of pressure reads (Emanuel 1994):

$$
\frac{\mathrm{d} T}{\mathrm{~d} p}=\frac{(1+r)}{\rho\left(c_{p \mathrm{~d}}+r c_{p \mathrm{v}}\right)}\left(1+\frac{L_{\mathrm{v}} r}{R_{\mathrm{d}} T}\right) /\left\{1+\frac{L_{\mathrm{v}}^{2} r(1+r / \varepsilon)}{R_{\mathrm{v}} T^{2}\left(c_{p \mathrm{~d}}+r c_{p \mathrm{v}}\right)}\right\},
$$

where $r$ is the mixing ratio $\left(=q_{\mathrm{wv}} /\left(1-q_{\mathrm{wv}}\right)\right)$, and the values for the parameters used in (11) are listed in Table 1. Air density $\rho$ in (10) and (11) is obtained by assuming 
TABLE 1. SUMMARY OF PARAMETERS USED IN THE RADIATIVE-CONVECTIVE MODEL

\begin{tabular}{ccc}
\hline Parameter & Definition & Value \\
\hline$c_{p \mathrm{~d}}$ & Heat capacity at constant pressure of dry air & $1005.7 \mathrm{~J} \mathrm{~kg}^{-1} \mathrm{~K}^{-1}$ \\
$c_{p \mathrm{v}}$ & Heat capacity at constant pressure of water vapour & $1870 \mathrm{~J} \mathrm{~kg}^{-1} \mathrm{~K}^{-1}$ \\
$L_{\mathrm{v}}$ & Latent heat of vaporization & $2.5 \times 10^{6} \mathrm{~J} \mathrm{~kg}^{-1}$ \\
$R_{\mathrm{d}}$ & Gas constant of dry air & $287.04 \mathrm{~J} \mathrm{~kg}^{-1} \mathrm{~K}^{-1}$ \\
$R_{\mathrm{v}}$ & Gas constant of water vapour & $468.5 \mathrm{~J} \mathrm{~kg}^{-1} \mathrm{~K}^{-1}$ \\
\hline
\end{tabular}

water vapour and dry air to be ideal gases, so it is a function of pressure, temperature and mixing ratio (i.e. $\rho=p(1+r) /\left\{T R_{\mathrm{d}}(1+r / \varepsilon)\right\}$ ). The tropospheric mixing ratio $r$ in (11) is obtained as follows. First, I assume a vertical profile of relative humidity $(R H)$ in the troposphere like that first introduced by Manabe and Wetherald (1967):

$$
R H=R H_{\mathrm{s}}\left(p / p_{\mathrm{s}}-0.02\right) / 0.98,
$$

where $R H_{\mathrm{S}}$ is the relative humidity at the surface. Then, I use the expression of the saturation vapour pressure $e_{\mathrm{s}}$ as a function of temperature (Bohren and Albrecht 1998):

$$
e_{\mathrm{S}}=e_{\mathrm{s} 0} \exp \left\{6808\left(\frac{1}{T_{0}}-\frac{1}{T}\right)-5.09 \ln \left(\frac{T}{T_{0}}\right)\right\},
$$

where $T_{0}(=273.15 \mathrm{~K})$ is a reference temperature, and $e_{\mathrm{s} 0}(=6.11 \mathrm{hPa})$ is the saturation vapour pressure at $T_{0}$. Since the partial pressure of water vapour $e$ is equal to $e_{\mathrm{S}} R H$, the mixing ratio $r$ is expressed as a function of $e$ through the ideal gas equations for dry air and water vapour (i.e. $\left.r=e_{\mathrm{S}} R H \varepsilon /\left(p-e_{\mathrm{S}} R H\right)\right)$. For tropospheric conditions, where the previous procedure gives mixing ratios lower than $3 \times 10^{-6},(12)$ is not used, and the threshold value $r_{\min }=3 \times 10^{-6}$ is applied. I assume a stratosphere in radiative equilibrium (i.e. satisfying (9)) with a constant $r$ equal to the tropopause value $r_{\text {tpp }}$.

The moist adiabatic lapse rate (11) for non-saturated air has been used in many studies (e.g. Kasting et al. 1984); it assumes that in descending air parcels the evaporation of water droplets or sublimation of ice crystals extracts heat energy from the air (see, e.g. Thomas and Stamnes 1999).

Finally, I also analyse the results obtained by assuming a saturated troposphere. In this case, the prescribed vertical temperature profile in the troposphere in terms of pressure follows (11) for saturation conditions (i.e. $R H=1=100 \%$ ) instead of (12). Note that now $e$ is directly given by (13), and $r=e_{\mathrm{s}} \varepsilon /\left(p-e_{\mathrm{s}}\right)$.

\section{(c) Solution procedure}

The solution procedure does not apply any angular approximation for the radiation field and is as follows:

(i) An arbitrary value of the tropopause level $p_{\text {tpp }}$ is chosen $\left(p_{\text {tpp }}>0.1 \mathrm{hPa}\right.$ to ensure LTE).

(ii) The tropospheric vertical temperature profile is obtained by integrating the tropospheric lapse rate (either (10) or (11)) from $p_{\mathrm{s}}$ to $p_{\mathrm{tpp}}$.

(iii) The stratospheric temperature profile is obtained by using the radiative equilibrium condition (9) applied to 100 points between $0.1 \mathrm{hPa}$ and $p_{\text {tpp }}$ (see appendix).

(iv) The difference is computed between the tropopause temperatures $T_{\text {tpp }}$ obtained from the third step (radiative-convective equilibrium condition) and from the fourth step (radiative equilibrium condition; see appendix). 


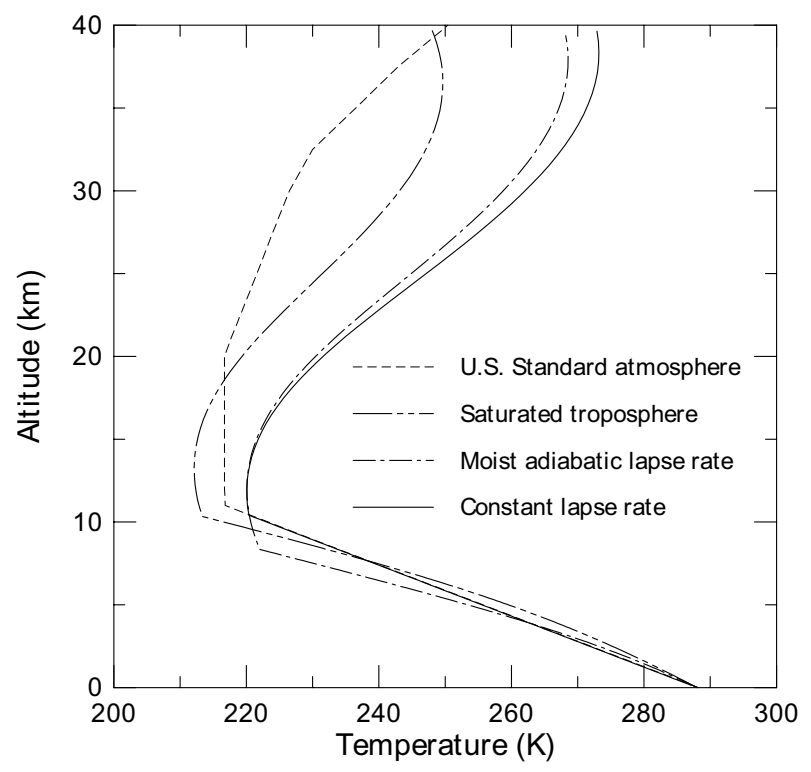

Figure 2. Temperature profiles in the non-grey atmosphere developed here. Both the constant lapse rate $\left(6.5 \mathrm{~K} \mathrm{~km}^{-1}\right.$; solid line) and the moist adiabatic (dash-dot line for a fixed profile of relative humidity; dashdot-dot line for a saturated troposphere) adjustments are used. The mass absorption coefficient in the atmospheric window is proportional to the partial pressure of water vapour (see text). The vertical profile of the US Standard Atmosphere (1976) is also shown.

(v) A new value of $p_{\text {tpp }}$ is chosen and steps (ii) to (v) are repeated until the difference in absolute value between radiative-convective $T_{\text {tpp }}$ and radiative $T_{\text {tpp }}$ is smaller than a threshold value (which is $10^{-3} \mathrm{~K}$ in the following results).

(vi) Note that the third step does not require a vertical discretization of the troposphere, since it is performed by applying a fifth-order Cash-Karp Runge-Kutta method with adaptive step size (Press et al. 1994). The fourth step is solved by discretizing the atmosphere into 250 levels including the surface (150 tropospheric plus 100 stratospheric; see appendix).

\section{EVALUATION OF THE RADIATIVE-EQUILIBRIUM MODEL}

Vertical temperature profiles for different convective adjustments are shown in Fig. 2. The mass absorption coefficient of water vapour outside the atmospheric window $k_{\mathrm{wV}}$, is equal to $0.3 \mathrm{~m}^{2} \mathrm{~kg}^{-1}$ in order to produce the observed optical thickness $(\approx 4.4$; see Goody and Yung 1989; Thomas and Stamnes 1999). The mass absorption coefficient of carbon dioxide $k_{\mathrm{cd}}\left(=0.05 \mathrm{~m}^{2} \mathrm{~kg}^{-1}\right)$ has been tuned to give a climate sensitivity of approximately $1.2 \mathrm{~K}$ to a doubling of the $\mathrm{CO}_{2}$ concentration, if nothing changes apart from the temperature (Houghton 1997). Finally, and since the continuum absorption within the atmospheric window is proportional to the partial pressure of water vapour $e$ (Houghton 1997), I use a mass absorption coefficient of water vapour inside the atmospheric window $k_{\mathrm{wvw}}=e k_{\mathrm{wvwe}}$, where the value of $k_{\mathrm{wvwe}}\left(2 \times 10^{-6} \mathrm{~m}^{2} \mathrm{~kg}^{-1} \mathrm{~Pa}^{-1}\right)$ has been tuned to give an opacity in the atmospheric window two orders of magnitude smaller than that observed outside the window for current conditions (see Kasting et al. 1984). A summary of the results for the three cases shown in Fig. 2 is listed in Table 2.

The stratospheric warming observed in Fig. 2 is due to the absorption by ozone. A greater value of the ozone mass absorption coefficient $k_{\mathrm{oz}}$, would imply a higher 
TABLE 2. PARAMETERS FOR THE MODEL ATMOSPHERES SHOWN IN FIG. 2

\begin{tabular}{lccc}
\hline & $\begin{array}{c}\text { Constant lapse rate } \\
\left(6.5 \mathrm{~K} \mathrm{~km}^{-1}\right)\end{array}$ & $\begin{array}{c}\text { Moist adiabatic } \\
\text { lapse rate }\end{array}$ & $\begin{array}{c}\text { Saturated } \\
\text { troposphere }\end{array}$ \\
\hline Surface temperature, $T_{\mathrm{S}}(\mathrm{K})$ & 288.0 & 288.0 & 288.0 \\
Outgoing long-wave radiation OLR $\left(\mathrm{W} \mathrm{m}^{-2}\right)$ & 295.8 & 298.7 & 271.6 \\
Surface convective flux, $F_{\mathrm{cv}}\left(\mathrm{W} \mathrm{m}^{-2}\right)$ & 191.0 & 195.6 & 175.2 \\
Tropopause height, $Z_{\mathrm{tpp}}(\mathrm{km})$ & 10.40 & 8.34 & 10.33 \\
Tropopause temperature, $T_{\mathrm{tpp}}(\mathrm{K})$ & 220.4 & 222.0 & 213.4 \\
\hline
\end{tabular}

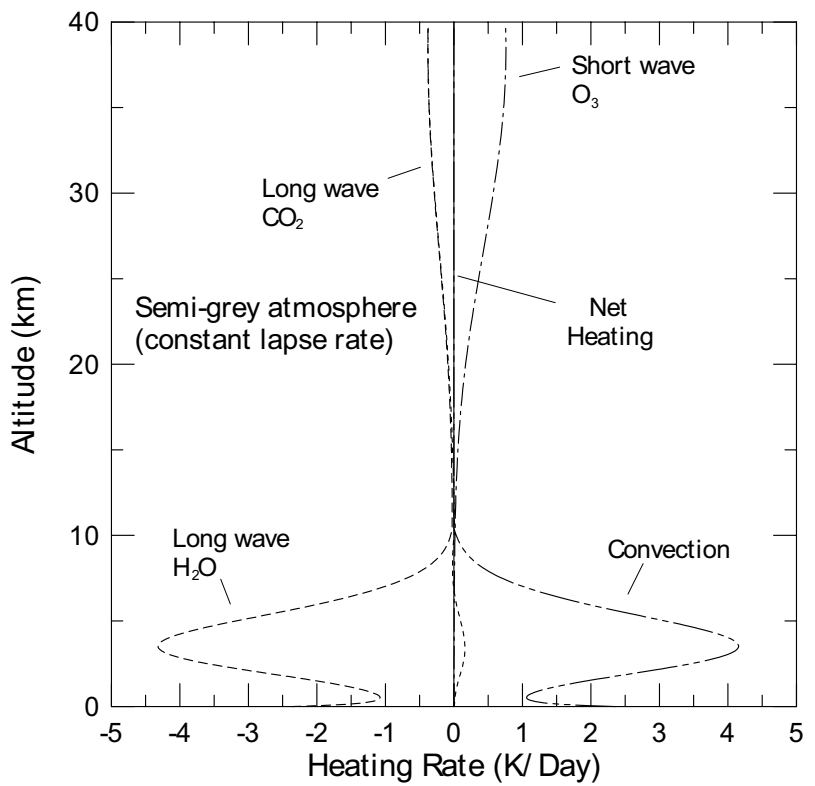

Figure 3. Vertical profile of heating rates in a non-grey atmosphere with a constant lapse rate $\left(6.5 \mathrm{~K} \mathrm{~km}^{-1}\right)$ adjustment (solid line in Fig. 2).

temperature in the higher layers shown in Fig. 2, but not a substantial warming near the tropopause. In (8), the solar irradiance at TOA integrated over the ozone band $S_{\mathrm{oz}}(0)$ is taken as $34 \mathrm{~W} \mathrm{~m}^{-2}$ ( $10 \%$ of the current incoming radiation divided by four), and is independent of the OLR. However, a more accurate model should include the dependence of $S_{\mathrm{oz}}(0)$ as a function of OLR, since stationary conditions implicitly assume $0.25 S(1-a)=$ OLR, $S$ being the solar constant and $a$ the planetary albedo (since OLR $\approx 296 \mathrm{~W} \mathrm{~m}^{-2}$ from Table 2 , and $0.25 S \approx 340 \mathrm{~W} \mathrm{~m}^{-2}$, a globally-averaged clear-sky albedo $a \approx 0.13$ is implicitly assumed). The absorption of short-wave radiation by ozone in the atmosphere is $\approx 4.3 \mathrm{~W} \mathrm{~m}^{-2}$, approximately half of the expected result $\left(\approx 9 \mathrm{~W} \mathrm{~m}^{-2}\right.$, as in Lindzen et al. (1982)). A higher value of $k_{\mathrm{oz}}$ would increase this value, though it would produce an unrealistic warming at high altitudes. In addition, the semi-grey approach seems to overestimate both OLR and convective flux at the surface $F_{\mathrm{cv}}$. For example, the standard RCM developed by Lindzen et al. (1982) gives an OLR $\approx 274 \mathrm{~W} \mathrm{~m}^{-2}$ and $F_{\mathrm{cv}} \approx 160 \mathrm{~W} \mathrm{~m}^{-2}$ (compare these with the values shown in Table 2).

Heating rates for the semi-grey atmosphere with a constant lapse rate, shown in Fig. 2, are depicted in Fig. 3. The maximum radiative cooling occurs at an altitude of about $3.5 \mathrm{~km}$ with a temperature near $265.0 \mathrm{~K}$. This level is close to the emission 
level, $Z_{\mathrm{el}}$, defined by Lindzen et al. (2001), which corresponds to the altitude where the black body emission at the atmospheric temperature $T_{\text {el }}$ coincides with the OLR $\left(T_{\mathrm{el}}=268.6 \mathrm{~K}\right.$ and $Z_{\mathrm{el}}=3.0 \mathrm{~km}$ for an OLR $\left.\approx 295 \mathrm{~W} \mathrm{~m}^{-2}\right)$.

A slight warming effect of carbon dioxide in the troposphere is also observed in Fig. 3, being in agreement with the results found by Lindzen et al. (1982) in a more elaborate model. This effect is due to the water vapour band overlapping the carbon dioxide band. Low values of heating rates due to ozone are found in the stratosphere (warming rate $\approx 0.8 \mathrm{~K} \mathrm{day}^{-1}$ at $38 \mathrm{~km}$ ). Stratospheric cooling rates by water vapour are similar to those for carbon dioxide, since simulations use a constant value of specific humidity in the stratosphere (equal to the tropopause value), overestimating its water vapour content.

\section{RESULTS}

\section{(a) Pure radiative equilibrium atmosphere}

The ground temperature $T_{\mathrm{g}}$, in pure radiative equilibrium atmospheres, differs from the air temperature at the surface $T_{\mathrm{S}}$ (see e.g. Goody and Yung 1989). Since we assume liquid-vapour equilibrium conditions, the surface mixing ratio equals the saturation value at $T_{\mathrm{s}}$. The mixing ratio at other atmospheric levels is set equal to the surface value, in consistency with the assumption applied to the stratosphere in our RCM (see the appendix). Note that this assumption does not lead to supersaturated layers in the model atmosphere, since the vertical temperature gradient obtained in the purely radiative atmosphere is small enough to keep $R H$ below the saturation point at all atmospheric levels. The model is solved following the numerical procedure applied to the stratosphere and detailed in the appendix (which is based on Arking and Grossman (1972)).

Figure 4 shows the OLR as a function of $T_{\mathrm{s}}$ (smaller than $T_{\mathrm{g}}$ ) for pure radiative equilibrium atmospheres with different opacities within the atmospheric window. The shortdashed line in Fig. 4 corresponds to an atmosphere with a mass absorption coefficient in the atmospheric window proportional to the partial pressure of water vapour $e$, as used in Figs. 2 and 3. Other cases use a constant value of the mass absorption coefficient within the atmospheric window (not realistic for earth's current atmosphere). Since the value of the mass absorption coefficient of the water vapour outside the window $k_{\mathrm{wv}}$, is $0.3 \mathrm{~m}^{2} \mathrm{~kg}^{-1}$, solutions in Fig. 4 range from the grey assumption $\left(k_{\mathrm{wvw}}=0.3 \mathrm{~m}^{2} \mathrm{~kg}^{-1}\right.$; solid line) to the totally transparent window $\left(k_{\mathrm{wvw}}=0.0 \mathrm{~m}^{2} \mathrm{~kg}^{-1}\right.$; dash-dot-dot line).

Note that the OLR is not a monotonic function on the surface temperature, since the effect of using a region of low absorption in the infrared spectrum is of particular importance in optically thick atmospheres (i.e. at high temperatures) but not in thin ones (i.e. at low temperatures). In Fig. 4 , I find a radiation limit $\approx 210 \mathrm{~W} \mathrm{~m}^{-2}$ (at $T_{\mathrm{S}} \approx 225 \mathrm{~K}$ ) when using a water vapour continuum absorption proportional to its partial pressure. This upper bound on the OLR appears to be due to the competition between the optical depth predicted by the radiative equilibrium condition and that by the liquid-vapour equilibrium condition (see the discussion in Nakajima et al. (1992)). Note that if the planetary atmosphere requires a long-wave emission greater than the radiation limit value (e.g. in order to balance the absorbed short-wave radiation), the liquid-vapour equilibrium condition does not hold and the liquid phase entirely evaporates. This runaway greenhouse effect was found by Simpson (1927), Komabayasi (1967) and Ingersoll (1969) in the analysis of grey atmospheres (as the solid line in Fig. 4) whose main absorbing gas is also in liquid phase at the surface. 


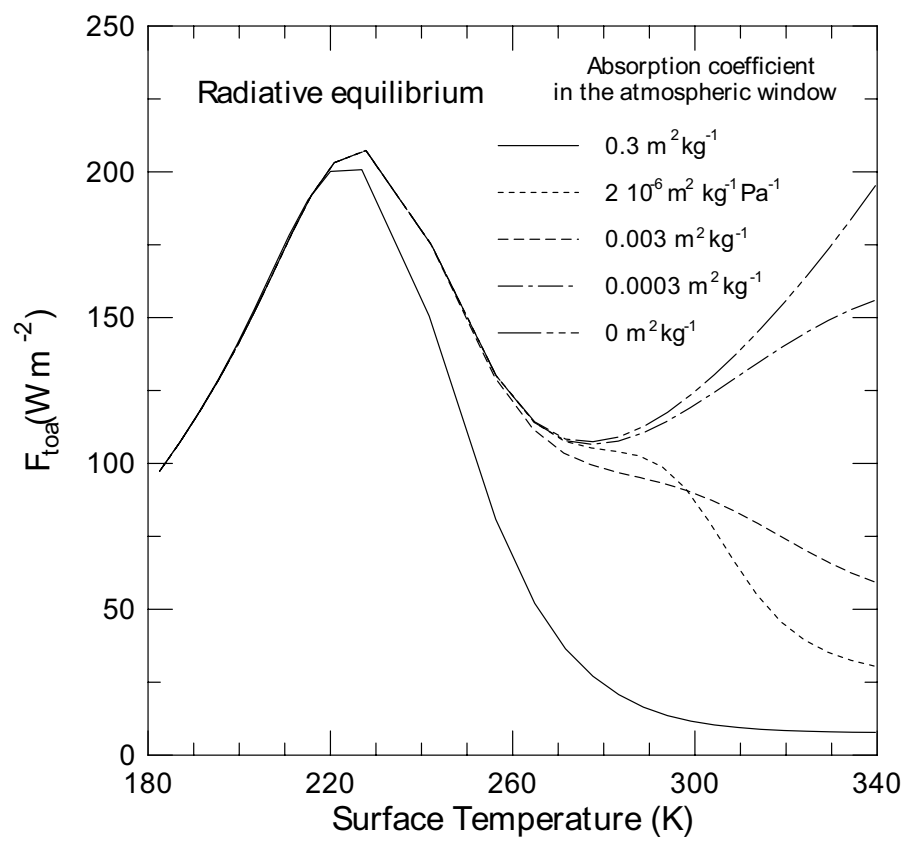

Figure 4. Long-wave radiation emitted at the top of the atmosphere $\left(F_{\text {toa }} \equiv \mathrm{OLR}\right)$ as a function of the surface temperature in non-grey atmospheres in pure radiative equilibrium for different values of the mass absorption coefficient of water vapour within the atmospheric window. The mass absorption coefficient outside the atmospheric window is constant $\left(0.3 \mathrm{~m}^{2} \mathrm{~kg}^{-1}\right)$. Within the window, the mass absorption coefficient is constant except for the short-dashed line, where it is proportional to the partial pressure of water vapour. The surface is also in vapour-liquid equilibrium.

Solutions shown in Fig. 4 reveal the existence of different values of $T_{\mathrm{S}}$ (i.e. of atmospheric temperature profiles) that emit the same amount of OLR (e.g. see the OLR $=150 \mathrm{~W} \mathrm{~m}^{-2}$ case). However, the steady states lying on branches with negative climate sensitivity (i.e. $\partial T_{\mathrm{S}} / \partial \mathrm{OLR}<0$ ) are unstable (unattainable and physically impossible) climates (e.g. North et al. 1981; Goody and Yung 1989). Then, non-grey atmospheres with a water vapour continuum absorption proportional to the partial pressure do not show a multiple equilibrium of stable states (see the short-dashed line in Fig. 4). In contrast, non-grey atmospheres with constant mass absorption coefficients with very weak (or null) absorption within the atmospheric window may show a multiple equilibrium of stable states (note the existence of two distinct branches with $\partial T_{\mathrm{S}} / \partial \mathrm{OLR}>0$ for the dash-dotted line in Fig. 4). In these cases, a secondary radiation limit at high temperatures (relative or absolute depending on the value of the mass absorption coefficient within the atmospheric window) is found.

Note that the result for an atmosphere with a window opacity similar to that for the earth's current state (short-dashed line in Fig. 4) does not show a multiple equilibrium of stable states. It implies that the multiplicity of stable states found in Fig. 4 is intrinsically different from that obtained in RCMs applied to earth's current state (Li et al. 1997; Rennó 1997; Ide et al. 2001). Indeed, the multiple equilibria found by $\mathrm{Li}$ et al. (1997) in a RCM is caused by the assumption that the surface albedo is a function of surface temperature, being very similar in nature to the multiple solutions found in vertically averaged, latitude-dependent energy-balance model with ice-albedo feedback (see, e.g. North et al. 1981). The multiple solutions of stable states of the mean standard 


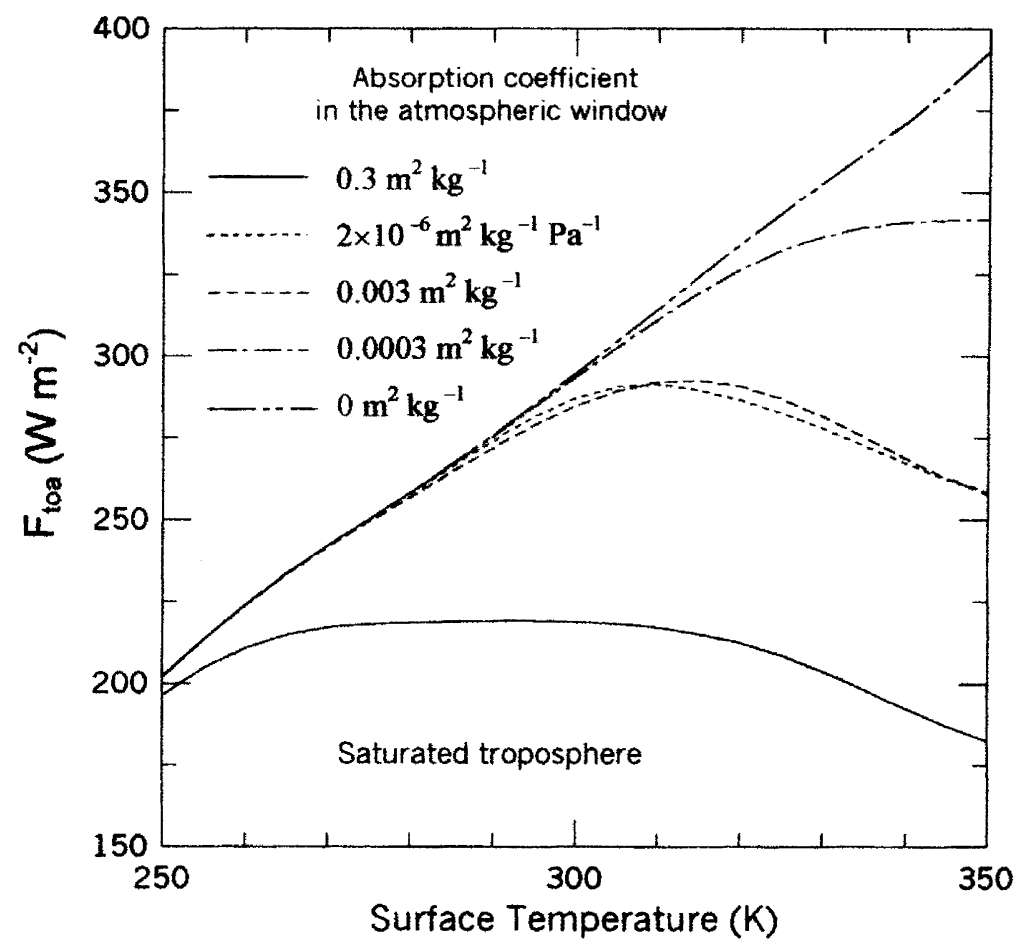

Figure 5. Long-wave radiation emitted at the top of the atmosphere $\left(F_{\text {toa }} \equiv\right.$ OLR) as a function of the surface temperature in radiative-convective non-grey atmospheres with fully saturated tropospheres for different values of the mass absorption coefficient of water vapour within the atmospheric window. The mass absorption coefficient outside the atmospheric window is constant $\left(0.3 \mathrm{~m}^{2} \mathrm{~kg}^{-1}\right)$. Within the window, the mass absorption coefficient is constant except for the short-dashed line, where it is proportional to the partial pressure of water vapour.

atmosphere found by Rennó (1997) arise when including a complete hydrological cycle, whereas Ide et al. (2001) obtained a bimodality in the solutions of a tropical (and a subtropical) atmosphere due to the competition between ascending and descending energy fluxes. Here, the multiple equilibria of the mean standard atmosphere arise from the non-grey absorption of infrared radiation only. This multiplicity of steady states has recently been analysed by employing $1 \mathrm{D}$ models with an angular approximation for the radiation field (Pujol and North 2002, 2003).

\section{(b) Radiative-convective atmosphere}

Figure 5 shows the OLR as a function of the surface temperature in a radiativeconvective atmosphere with a troposphere fully saturated, for different values of the absorption coefficient within the atmospheric window. Grey and non-grey cases have been extensively analysed by several investigators (see e.g. Nakajima et al. 1992; Pujol and North 2002) using model atmospheres with angular approximations for the radiation field. In contrast with the results obtained in previous studies, here we find that the exact method predicts a radiation limit in radiative-convective atmospheres that exceeds that predicted from pure radiative equilibrium atmospheres (e.g. compare the maximum value of OLR reached by the short-dashed line in Figs 4 and 5). In addition, we find that convection removes the stable state found at high values of surface temperature in a pure radiative equilibrium atmosphere (e.g. compare the behaviour of the dash-dotted lines in Figs. 4 and 5). Note that here we analyse profiles with surface temperatures lower than 


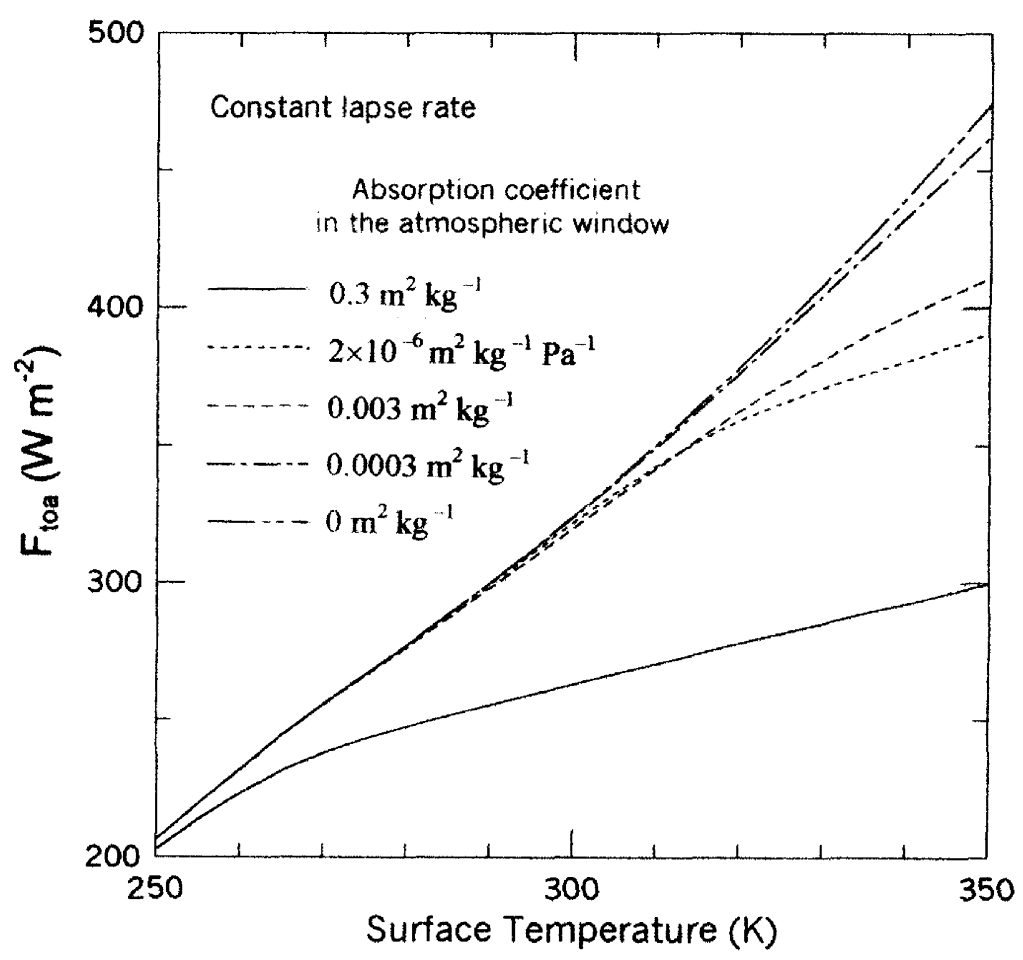

Figure 6. As in Fig. 5 except for a troposphere with a constant lapse rate $\left(6.5 \mathrm{~K} \mathrm{~km}^{-1}\right)$ and a relative-humidity profile decreasing with height (see (12)).

$350 \mathrm{~K}$, since the assumption of water vapour as an ideal gas breaks down at high surface pressures (reached at high surface temperatures; see e.g. Kasting (1988)). However, the extrapolation of the assumption of water vapour as an ideal gas for higher values of surface temperatures (not shown here) does not reveal the existence of a multiplicity of stable states.

Figure 6 is similar to Fig. 5 but for a troposphere with a constant lapse rate $\left(\Gamma_{\mathrm{e}}=6.5 \mathrm{~K} \mathrm{~km}^{-1}\right)$ and $R H$ decreasing with pressure as in (12). Here, and differing from the fully saturated troposphere analysed in Fig. 5, we do not find any radiation limits. Note that the variable $R H$ profile with a constant lapse-rate adjustment (as in Fig. 6) is representative of mean hemispheric conditions, whereas a uniform $R H$ profile has been used to simulate warm and moist tropical pools (see Pierrehumbert 1995). Complete saturation profiles (as in Fig. 5) have been assumed in the analysis of much hotter scenarios, where the variable $R H$ profile would predict an inconsistent low amount of water vapour at high altitudes.

The variable $R H$ profile for the case with water vapour continuum absorption within the atmospheric window proportional to the partial pressure of water vapour, shown in Fig. 6, emits without bound (which differs from the result found by Vardavas and Carver 1985). In comparison, a uniform $R H$ profile ( $=80 \%$ throughout the entire troposphere) with a constant lapse-rate adjustment $\left(\Gamma_{\mathrm{e}}=6.5 \mathrm{~K} \mathrm{~km}^{-1}\right)$ reaches an asymptotic SKI limit (atmospheric radiation limit, see Pujol and North (2003)) approximately equal to $315 \mathrm{~W} \mathrm{~m}^{-2}$ (not shown), being very similar to that obtained by Pierrehumbert (1995) for the same conditions using the National Center for Atmospheric Research (NCAR) 
Community Climate Model CCM2 radiation code $\left(\approx 320 \mathrm{~W} \mathrm{~m}^{-2}\right)$. The convective adjustment with a moist adiabatic lapse rate (not shown here) does not alter this threshold value excessively $\left(\approx 304 \mathrm{~W} \mathrm{~m}^{-2}\right)$, although it is reached at intermediate temperatures (differing from Vardavas and Carver (1985) but in agreement with Nakajima et al. (1992)). I point out that the atmosphere with a saturated troposphere (Fig. 5) behaves similarly to the moist adiabatic case with a uniform $R H(<100 \%)$ profile in the troposphere. Since it is more opaque, the SKI limit is smaller $\left(\approx 291 \mathrm{~W} \mathrm{~m}^{-2}\right.$; see the short-dashed line in Fig. 5).

The multiplicity of stable states does not arise in the RCM developed here, since the tropopause temperature always lies on the stable branch of low temperatures shown in Fig. 4. This means that very high values of surface temperature imply very high tropopause levels (which imply relatively low values of the tropopause temperature). Thus, the tropopause temperature as a function of the OLR does not behave like Fig. 4. Note that the tropopause temperature in a RCM with non-zero non-grey absorption does behave as in Fig. 4 when using Eddington's approximation for describing the radiation field and assuming monochromatic radiative equilibrium conditions in a stratosphere transparent to sunlight (see Pujol and North 2003). The importance of solving the radiative-convective atmosphere by using the exact method described in the appendix is that I assume total radiative equilibrium conditions in the stratosphere (i.e. net shortwave equals net long-wave radiation).

\section{CONClusions}

The aim of this paper has been to analyse the effects of convective processes on the multiple equilibria of stable states found in radiative equilibrium models. For this purpose I have developed a 1D RCM with a very simple non-grey absorption and a convective adjustment to simulate the mean standard atmosphere. The model assumes uniform values of the mass absorption coefficient in finite width spectral bands. The main novelty in comparison with previous studies (Nakajima et al. 1992; Pujol and Fort 2002; Pujol and North 2002, 2003) is that I do not apply any angular approximation for the radiation field. As discussed above, it allows for applying total (i.e. broad-band) radiative equilibrium conditions in the stratosphere which are far more realistic (and less restrictive) than monochromatic radiative equilibrium conditions (as in Pujol and North 2002, 2003). The gases absorbing infrared radiation in the model are water vapour and carbon dioxide. Sunlight is absorbed by atmospheric ozone only. However, the structure of the model permits further refinements in terms of including more short-wave as well as long-wave absorption gases. The model has been calibrated to give the expected sensitivity to changes in carbon dioxide as well as to produce the optical thickness observed for the earth's current mean standard atmosphere.

A pure radiative equilibrium version of the model atmosphere developed here, with very weak absorption within the atmospheric window, shows multiple equilibria of stable states. Such a multiplicity of stable states arises from the non-grey absorption only, since cases with weak to high absorption within the atmospheric window give a single stable state (as for current values of the earth's atmospheric opacity). Therefore, it differs from the multiple equilibria in RCMs found by Li et al. (1997), Rennó (1997), Lorenz et al. (1999) and Ide et al. (2001). Such a multiplicity of stable states has been analysed by Pujol and Fort (2002) and Pujol and North $(2002,2003)$ in planetary atmospheres, and solved with an angular approximation for the radiation field and a stratosphere in monochromatic radiative equilibrium. 
I have found that the introduction of a convective regime (either a fully saturated troposphere or a constant lapse-rate adjustment with a variable $R H$ profile) removes the multiple equilibria of stable solutions found in pure radiative equilibrium models. Note that this result differs from the multiple equilibria of stable states found in a simple non-grey RCM developed by Pujol and North (2002). That study made use of Eddington's approximation for the radiation field, which led to an analytical solution for the stratospheric vertical temperature profile assumed to be in monochromatic radiative equilibrium. As already pointed out, the solution of the radiation field without using any angular approximation as used here, avoids the very restrictive assumption of a monochromatic radiative equilibrium condition needed to quasi-analytically solve the RCM developed by Pujol and North (2002). This result clearly indicates the importance of using RCMs (though not those extremely simplified) instead of pure radiative models to study the behaviour of planetary atmospheres (e.g. to investigate the existence of the hysteresis cycle suggested by simple radiative equilibrium models).

Although the results found here contradict those obtained by Pujol and North (2002, 2003), who made use of a similar model for the same purpose of simulating the mean standard atmosphere, they do not necessarily invalidate the multiplicity of stable states observed in other purely radiative equilibrium model atmospheres (e.g. Li et al. 1997; Ide et al. 2001) developed with the aim of investigating a different aspect of the atmosphere. However, the results here may suggest that conclusions about the multiplicity of stable states and the bimodality of the climate system deduced from pure radiative equilibrium models (e.g. Ide et al. 2001) should be revisited by using elaborate RCMs which, depending on the study carried out, should also include parametrizations for large-scale dynamical processes.

\section{ACKNOWLEDGEMENTS}

The author gratefully acknowledges the support of a Fulbright-Generalitat de Catalunya postdoctoral grant, as well as the partial support of the Ministerio de Ciencia y Tecnología of the Spanish Government under contract REN 2000-1621 CLI.

\section{APPENDIX}

\section{Exact solution}

The condition of radiative equilibrium in the stratosphere (9) can be written as

$$
\sum_{m=1}^{5} \widetilde{h}_{m}(p)+\widetilde{h}_{\mathrm{SW}}(p)=0,
$$

where $\widetilde{h}_{m}=h_{m} / \rho$ and $\widetilde{h}_{\mathrm{SW}}=h_{\mathrm{SW}} / \rho$ with $\rho$ the air density at pressure $p\left(\leqslant p_{\text {tpp }}\right)$ and ${\underset{h}{h}}_{m}$ as defined in (5). From (7), $\widetilde{h}_{\mathrm{SW}}$ is a function of the local pressure only. However, $\widetilde{h}_{m}$ depends on the temperature at any other vertical level, since (5) (divided by $\rho$ ) reads

$$
\begin{aligned}
\widetilde{h}_{m p}= & 2 \pi k_{m_{i}} q_{i p}\left(\beta_{m p_{\mathrm{s}}} B_{p_{\mathrm{s}}} E_{2}\left(x_{\mathrm{s}}\right)+\frac{1}{g} \int_{p}^{p_{\mathrm{s}}} \beta_{m p^{\prime}} B_{p^{\prime}} E_{1}\left(x_{u}\right) k_{m_{i}} q_{i p^{\prime}} \mathrm{d} p^{\prime}\right. \\
& \left.+\frac{1}{g} \int_{0}^{p} \beta_{m p^{\prime}} B_{p^{\prime}} E_{1}\left(x_{d}\right) k_{m_{i}} q_{i p^{\prime}} \mathrm{d} p^{\prime}-2 \beta_{m p} B_{p}\right),
\end{aligned}
$$


where $\widetilde{h}_{m p}=\widetilde{h}_{m}(p), q_{i p}=q_{i}(p), \beta_{m p}=\beta_{m}(p), B_{p}=B(p)$ and $E_{1,2}$ are exponential functions with arguments

$$
x_{\mathrm{s}}=\frac{1}{g} \int_{p}^{p_{\mathrm{s}}} k_{m i} q_{i p^{\prime}} \mathrm{d} p^{\prime}, \quad x_{u}=\frac{1}{g} \int_{p}^{p^{\prime}} k_{m i} q_{i p^{\prime \prime}} \mathrm{d} p^{\prime \prime}, \quad x_{\mathrm{d}}=\frac{1}{g} \int_{p^{\prime}}^{p} k_{m i} q_{i p^{\prime \prime}} \mathrm{d} p^{\prime \prime} .
$$

Equation (A.2) follows from (5) using (4a) and (4b), and the definition of the exponential functions (e.g. Goody and Yung 1989). Following Arking and Grossman (1972), the two integrals in (A.2) can be expressed in terms of $E_{2}$ by using one property of the exponential functions (i.e. $\mathrm{d} E_{2}(x) / \mathrm{d} x=-E_{1}(x)$ ). Then, I divide the atmosphere into $N$ vertical layers $(N+1$ atmospheric temperatures, where subscripts 1 refer to the surface and $N+1$ to the TOA). As a result, a simple discrete approximation of (A.2) evaluated at the level $n$ reads:

$$
\begin{aligned}
\widetilde{h}_{m(n)}= & 2 \pi k_{m_{i}} q_{i(n)}\left(\beta_{m(1)} B_{1} E_{2 u(1)}+\frac{1}{2} \sum_{l=1}^{n-1}\left(\beta_{m(l+1)} B_{l+1}+\beta_{m(l)} B_{l}\right)\left(E_{2 u(l+1)}-E_{2 u(l)}\right)\right. \\
& \left.+\frac{1}{2} \sum_{l=n}^{N}\left(\beta_{m(l+1)} B_{l+1}+\beta_{m(l)} B_{l}\right)\left(E_{2 d(l+1)}-E_{2 d(l)}\right)-2 \beta_{m(n)} B_{n}\right),
\end{aligned}
$$

where

$$
E_{2 u(l)}=E_{2}\left\{\frac{1}{2 g} \sum_{s=l}^{n-1}\left(k_{m i} q_{i(s+1)}+k_{m i} q_{i(s)}\right)\left(p_{\mathrm{s}}-p_{s+1}\right)\right\},
$$

and

$$
E_{2 d(l)}=E_{2}\left\{\frac{1}{2 g} \sum_{s=n}^{l-1}\left(k_{m i} q_{i(s+1)}+k_{m i} q_{i(s)}\right)\left(p_{\mathrm{s}}-p_{s+1}\right)\right\} .
$$

In (A.4) I assume continuity of temperatures at the surface (i.e. $T_{\mathrm{g}}=T_{\mathrm{s}}$ ). I also use $\widetilde{h}_{m(l)}=\widetilde{h}_{m}\left(p_{l}\right), q_{i(l)}=q_{i}\left(p_{l}\right), \beta_{m(l)}=\beta_{m}\left(p_{l}\right)$, and $B_{l}=B\left(p_{l}\right)$ where $p_{l}$ is the pressure at the level $l(1 \leqslant l \leqslant N+1)$. Note that I have $N+1$ equations for $\widetilde{h}_{m(n)}$ with $N+1$ atmospheric temperature values (i.e. $N+1$ values of $B$ ). This set of linear (in $B$ ) algebraic equations can be written as

$$
\widetilde{\mathbf{h}}_{m}=\mathcal{A}_{m} \mathbf{B},
$$

where $\widetilde{\mathbf{h}}_{m}$ and $\mathbf{B}$ are vectors of dimensions $N+1$, and $\boldsymbol{A}_{m}$ is a matrix of dimensions $(N+1) \times(N+1)$, whose coefficients are obtained from the $N+1$ equations similar to (A.4). Equation (A.7) expressed in index form is $\widetilde{h}_{m}^{i}=\sum_{j=1}^{N+1} A_{m}^{i j} B^{j}$ for $1 \leqslant i \leqslant$ $N+1$.

Let us assume that the tropopause is at the level $t$ (i.e. $p_{t}=p_{\text {tpp }}$ ). Then, equation (A.7) for stratospheric values $(i>t)$ is $\widetilde{h}_{m}^{i}=\sum_{j=t+1}^{N+1} A_{m}^{i j} B^{j}+g_{m}^{i}$, where $g_{m}^{i}=$ $\sum_{j=1}^{t} A_{m}^{i j} B^{j}$ and $t+1 \leqslant i \leqslant N+1$. Note that the vector $g_{m}^{i}$ is known since the tropospheric lapse rate is integrated from the surface (at $p_{\mathrm{S}}$ and $T_{\mathrm{s}}$, which are prescribed values) to the tropopause (at $p_{\text {tpp }}$, which is a 'guess' value) using either (10) or (11). Then, the radiative equilibrium condition for the stratosphere expressed in matrix form reads

$$
\sum_{m=1}^{5} \widetilde{\mathbf{h}}_{m}+\mathbf{h}_{\mathrm{SW}}=\sum_{m=1}^{5} \mathcal{A}_{m} \mathbf{B}+\sum_{m=1}^{5} \mathbf{g}_{m}+\mathbf{h}_{\mathrm{SW}}=0,
$$


TABLE A.1. Polynomial FITS AS A FUNCTION OF TEMPERATURE $(T)$ TO THE EFFECTIVE WIDTH OF THE ATMOSPHERIC WINDOWS FOR BANDS $2\left(\beta_{2}\right), 3\left(\beta_{3}\right), 4\left(\beta_{4}\right)$ AND $5\left(\beta_{5}\right)$ (SEE FIG. 1)

\begin{tabular}{ccc}
\hline Band & $100 \mathrm{~K} \leqslant T<200 \mathrm{~K}$ & $200 \mathrm{~K} \leqslant T \leqslant 600 \mathrm{~K}$ \\
\hline 2 & $0.1982-0.00425577 T$ & $-0.278986-2.5265 \times 10^{-5} T+2.41602 \times 10^{-5} T^{2}$ \\
$(8-12 \mu \mathrm{m})$ & $+2.71143 \times 10^{-5} T^{2}$ & $-9.51274 \times 10^{-8} T^{3}+1.37678 \times 10^{-10} T^{4}$ \\
& $-3.84894 \times 10^{-8} T^{3}$ & $-7.05795 \times 10^{-14} T^{5}$ \\
3 & $0.07557-0.001921 T$ & $-0.280979+0.00350219 T-1.35706 \times 10^{-5} T^{2}$ \\
$(12-13 \mu \mathrm{m})$ & $+1.49635 \times 10^{-5} T^{2}$ & $+2.49572 \times 10^{-8} T^{3}-2.2607 \times 10^{-11} T^{4}$ \\
& $-3.08826 \times 10^{-8} T^{3}$ & $+8.14011 \times 10^{-15} T^{5}$ \\
4 & $0.0535941-0.00341667 T$ & $-0.960853+0.0145298 T-6.69421 \times 10^{-5} T^{2}$ \\
$(13-17.6 \mu \mathrm{m})$ & $+4.26572 \times 10^{-5} T^{2}$ & $+1.45634 \times 10^{-7} T^{3}-1.55659 \times 10^{-10} T^{4}$ \\
& $-1.09192 \times 10^{-7} T^{3}$ & $+6.60473 \times 10^{-14} T^{5}$ \\
5 & $0.191093+0.0162542 T$ & $1.69538-0.00786896 T+1.35115 \times 10^{-5} T^{2}$ \\
$(>17.6 \mu \mathrm{m})$ & $-0.000115921 T^{2}$ & $-8.1818 \times 10^{-9} T^{3}$ \\
& $+2.24399 \times 10^{-7} T^{3}$ & \\
\hline
\end{tabular}

where, now, $\widetilde{\mathbf{h}}_{m}, \mathbf{B}, \mathbf{g}_{m}$ and $\mathbf{h}_{\mathrm{SW}}$ are vectors of dimensions $N+1-t$ (stratospheric values) and $\boldsymbol{A}_{m}$ a matrix of dimensions $(N+1-t) \times(N+1-t)(m$ varies from 1 to 5 in agreement with Fig. 1). The vector $\mathbf{h}_{\mathrm{SW}}$ is obtained from (8). Since $\mathbf{h}_{\mathrm{SW}}, \mathbf{g}_{m}$ and $\boldsymbol{A}_{m}$ are independent of the stratospheric temperature, (A.8) can be solved by inverting the matrix $\sum_{m=1}^{5} \mathcal{A}_{m}$, from which the vector $\mathbf{B}$ (i.e. stratospheric temperatures) is determined. Since the tropopause is in both radiative-convective and radiative equilibrium, the level $p_{t+1}$ is equal to the level $p_{t}$. Then, I choose the tropopause level $p_{t}$ such that the temperature at level $p_{t+1}$ (obtained from (A.8)) is equal to the temperature at $p_{t}$, which is obtained from the vertical integration of the lapse rate (for numerical purposes, a difference smaller than $10^{-3} \mathrm{~K}$ in absolute value is used).

Note that the coefficients of the matrix $\mathcal{A}_{m}$ are not strictly independent of $\mathbf{B}$, since the effective width of the infrared bands $\beta_{m}$ are a function of $T$. The effect of using an effective width as a function of the local temperature is not very important at high altitudes (i.e. in the stratosphere), since the amount of infrared absorber there is very low. Therefore, I use a constant value of $\beta_{m}$ in the stratosphere, being equal to the tropopause value (i.e. the coefficients $\mathcal{A}_{m}$ in (A.8) are independent of B). In the troposphere, $\beta_{m}$ is expressed as a polynomial on temperature obtained by fitting (7) (i.e. the coefficients $\mathcal{A}_{m}$ in $\mathbf{g}_{m}$ are a function of $\left.\mathbf{B}\right)$. For the five infrared spectral bands shown in Fig. 1, the coefficients $c_{n}$ of the fitted polynomials are listed in Table A.1.

Arking, A. and Grossman, K.

Bohren, C. F. and Albrecht, B. A.

Emanuel, K. A.

Goody, R. M. and Yung, Y. L.

Houghton, J. T.

Ide, K., Le Treut, H., Li, Z.-X. and Ghil, M.

\section{REFERENCES}

1972 The influence of line shape and band structure on temperatures in planetary atmospheres. J. Atmos. Sci., 29, 937-949

1998 Atmospheric thermodynamics. Oxford University Press, New York, USA

1994 Atmospheric convection. Oxford University Press, New York, USA

1989 Atmospheric radiation: Theoretical basis. Second edition. Oxford University Press, New York, USA

1986 The physics of atmospheres. Second edition. Cambridge University Press, New York, USA

1997 Global warming. Second edition. Cambridge University Press, New York, USA

2001 Atmospheric radiative equilibria. Part II: Bimodal solutions for atmospheric optical properties. Clim. Dyn., 18, 29-49 
Ingersoll, A. P.

Jursa, A. S. (Ed.)

1969

1985

Kasting, J. F.

Kasting, J. F., Pollack, J. B. and Ackerman, T. P.

Komabayasi, M.

Li, Z.-X., Ide, K., Le Treut, H. and Ghil, M.

Lindzen, R. S., Hou, A. Y. and Farrell, B. F.

Lindzen, R. S., Chou, M.-D. and Hou, A. Y.

Lorenz, R. D., McKay, C. P. and Lunine, J. I.

Manabe, S. and Möller, F.

Manabe, S. and Strickler, R. F.

Manabe, S. and Wetherald, R. T.

Nakajima, S., Hayashi, Y.-Y. and Abe, Y.

North, G. R., Cahalan, R. F. and Coakley, J. A. Jr.

Pierrehumbert, R. T.

Press, W. H., Teukolsky, S. A., Vetterling, W. T. and

Flannery, B. P.

Pujol, T. and Fort, J.

Pujol, T. and North, G. R.

Rennó, N. O.

Simpson, G. C.

Thomas, G. E. and Stamnes, K.

Vardavas, I. M. and Carver, J. H.

Weaver, C. P. and Ramanathan, V.
The runaway greenhouse: A history of water on Venus. J. Atmos. Sci., 26, 1191-1198

'Handbook of geophysics and the space environment'. Air Force Geophysics Laboratory, Air Force systems command, United States Air Force

1988 Runaway and moist greenhouse atmospheres and the evolution of earth and Venus. Icarus, 74, 472-494

1984 Response of earth's atmosphere to increases in solar flux and implications for loss of water from Venus. Icarus, 57, 335355

1967 Discrete equilibrium temperatures of a hypothetical planet with the atmosphere and the hydrosphere of a one componenttwo phase system under constant solar radiation. J. Meteorol. Soc. Jpn, 45, 137-139

1997 Atmospheric radiative equilibria in a simple column model. Clim. Dyn., 13, 429-440

1982 The role of convective model choice in calculating the climate impact of doubling $\mathrm{CO}_{2}$. J. Atmos. Sci., 39, 1189-1205

2001 Does the earth have an adaptive infrared iris? Bull. Am. Meteorol. Soc., 82, 417-432

1999 Analytic investigation of climate stability on Titan: Sensitivity to volatile inventory. Planet. Space Sci., 47, 1503-1515

1961 On the radiative equilibrium and heat balance of the atmosphere. Mon. Weather Rev., 89, 503-532

1964 Thermal equilibrium of the atmosphere with a convective adjustment. J. Atmos. Sci., 21, 361-384

1967 Thermal equilibrium of the atmosphere with a given distribution of relative humidity. J. Atmos. Sci., 24, 241-259

1992 A study on the 'runaway greenhouse effect' with a one-dimensional radiative-convective equilibrium model. J. Atmos. Sci., 49, 2256-2266

1981 Energy balance climate models. Rev. Geophys. Space Phys., 19, 1191-1211

1995 Thermostats, radiator fins, and the local runaway greenhouse. J. Atmos. Sci., 52, 1784-1806

1994 Numerical recipes in Fortran. The art of scientific computing. Second edition. Cambridge University Press, New York, USA

2002 The effect of atmospheric absorption of sunlight on the runaway greenhouse point. J. Geophys. Res., 107D, 21, 4566

2002 Runaway greenhouse effect in a semigray radiative-convective model. J. Atmos. Sci., 59, 2801-2810

2003 Analytical investigation of the atmospheric radiation limits in semigray atmospheres in radiative equilibrium. Tellus, $\mathbf{5 5} \mathbf{A}$, 328-337

1997 Multiple equilibria in radiative-convective atmospheres. Tellus, 49A, 423-438

1927 Some studies in terrestrial radiation. Mem. R. Meteorol. Soc., 16, 69-95

1999 Radiative transfer in the atmosphere and ocean. Cambridge University Press, New York, USA

1984 Solar and terrestrial parameterizations for radiative-convective models. Planet. Space Sci., 32, 1307-1325

1985 Atmospheric temperature responses to variations in $\mathrm{CO}_{2}$ concentration and the solar-constant. Planet. Space Sci., 33, 11871207

1995 Deductions from a simple climate model: Factors governing surface temperature and atmospheric thermal structure. J. Geophys. Res., 100, 11585-11591 